\title{
Robots in Education and Care of Children with Developmental Disabilities: A Study on Acceptance by Experienced and Future Professionals
}

\author{
Daniela Conti $^{1,2} \cdot$ Santo Di Nuovo $^{2} \cdot$ Serafino Buono $^{3} \cdot$ Alessandro Di Nuovo $^{4,5}$ (D)
}

Accepted: 4 February 2016 / Published online: 1 June 2016

(c) The Author(s) 2016. This article is published with open access at Springerlink.com

\begin{abstract}
Research in the area of robotics has made available numerous possibilities for further innovation in the education of children, especially in the rehabilitation of those with learning difficulties and/or intellectual disabilities. Despite the scientific evidence, there is still a strong scepticism against the use of robots in the fields of education and care of people. Here we present a study on the acceptance of robots by experienced practitioners (specialized in the treatment of intellectual disabilities) and university students in psychology and education sciences (as future professionals). The aim is to examine the factors, through the Unified Theory of Acceptance and Use of Technology (UTAUT) model, that may influence the decision to use a robot as an instrument
\end{abstract}

The work of Alessandro Di Nuovo was partially supported by the EU under FP7 Grants no. 288899 (ROBOT-ERA).

Alessandro Di Nuovo

alessandro.dinuovo@unikore.it

Daniela Conti

danielaconti@unict.it

Santo Di Nuovo

s.dinuovo@unict.it

Serafino Buono

fbuono@oasi.en.it

1 Doctoral School in Neuroscience, University of Catania, Via SantaSofia 78, 95123 Catania, Italy

2 Department of Education Sciences, University of Catania, Via Biblioteca 4, 95124 Catania, Italy

3 Psychology Operative Unit, IRCCS "Maria SS" Oasi di Troina, 73, Conte Ruggero, 94018 Troina, Italy

4 Faculty of Engineering and Architecture, University of Enna Kore, Viale delle Olimpiadi, 94100 Enna, Italy

5 Department of Computing, Sheffield Hallam University, 153 Arundel Street, S1 2NU Sheffield, UK in the practice. The overall results confirm the applicability of the model in the context of education and care of children, and suggest a positive attitude towards the use of the robot. The comparison highlights some scepticism among the practitioners, who perceive the robot as an expensive and limited tool, while students show a positive perception and a significantly higher willingness to use the robot. From this experience, we formulate the hypothesis that robots may be accepted if more integrated with standard rehabilitation protocols in a way that benefits can outweigh the costs.

Keywords Socially assistive robotics - Rehabilitation and therapeutic tools - Unified theory of acceptance and use of technology (UTAUT) · Practitioners acceptance

\section{Introduction}

Recent technology advances and research achievements have boosted the area of robotics and resulted in a fast grow of possible applications, with a concrete impact on the everyday life of the common people. Among the applications in social contexts those regarding education and care of children have received particular attention thanks to evidence from several studies and more affordable new robotic platforms.

Despite the scientific success and increasing evidence and applications, it seems that the majority of people is still sceptical or even against the application of robots in real contexts like education and care of children. For example, according to a recent survey [1], European respondents indicated a positive attitude towards robots, but $60 \%$ of them said that robots should be banned from the area of care for children, the elderly and the disabled. Furthermore, only $3 \%$ said that robots in education are a priority, while $34 \%$ specifically stated that they should be banned from education. This is 
perhaps related to the common perception of robots as technically powerful but dangerous machines, which are mainly useful in space exploration, in military applications and in industry where there are no human beings around or just the ones that are employed to control them. We must say that the survey was very general and participants were given only very limited information about the concept of robots and how they could be used. The European study [2] did not elaborate on the term disability; therefore, it seems reasonable to expect that the negative sentiment is bigger the more the disabled person is seen to be cognitively compromised [3].

Public perception of an emerging scientific and technological product is important for the acceptance of such a product. Ethical studies based on public surveys toward using robots in eldercare and other applications showed a high acceptance for pet-like therapeutic robot, the human-like care robot, and a surveillance care robot [4]. But it also reported a rejection in the case of a bathing robot because of the judgment that the robot-based action would be inferior to the human-based action and that it would take away jobs from human workers. However at the same time, social robots are seen as a possible way to address the human resource and economic pressures on health care systems, but several studies stressed that care workers assisting the elderly should not be replaced by robots $[5,6]$.

A Swedish study [7] concluded that "people were globally positive towards the idea of intelligent service robots, which were seen as "domestic machines" that can be "controlled" and do mainly household tasks". In fact, Dautenhahn et al. [8] found that people would want a companion robot more as a servant (e.g. $96.4 \%$ wanted the robot to be able to do household jobs), but not a friend whereby young people were more inclined toward the friend role (i.e. $10.7 \%$ of participants wanted the robot to be able to look after their children).

However, this negative attitude is one of the biggest challenges that scientific research must address to be successful in giving real benefits in the field of education and care of children. To fill this gap, there have been many studies on the factors that can influence the acceptance by potential users and on how it can be increased. Among the objectives of current robotics research is the adaptation of the robot appearance and behaviours in order to improve the acceptance by the user $[9,10]$. Taking into account this scenario, we focus our research on the acceptance by practitioners that work on a daily basis with children with intellectual disabilities, and on a group of university students from two classes concerned with social professions: education and psychology.

The study was conducted using a platform for Socially Assistive Robotics (SAR) and a questionnaire based on the Unified Theory of Acceptance and Use of Technology (UTAUT) model. The objectives of the research are to confirm the reliability of the UTAUT model and its applicability in the context of education and care of children, and to evaluate the acceptance of robotics by individuals who are currently working in, or will work in the future, in the education of children with developmental disabilities. In particular, we aim to identify the factors that may influence the actual use of robotics in their profession, because we believe that this will greatly contribute to the design of robots and their actual application in social contexts.

The paper is organized as follows: Sect. 2 presents the scientific background and Sect. 3 presents the material and methods adopted in our research. In particular, after a brief description of the SAR field and the technology acceptance model used, Sect. 3 details the sample of practitioners and students interviewed, the robotic platform and the procedures followed to carry out the experiment and to analyse the data. Section 4 shows the results of the analyses about the acceptance by participants interviewed with a particular emphasis on the comparison between the two groups considered. Section 5 discusses the results and gives our explanation in relation to previous literature. Finally, Sect. 6 gives the conclusion and presents the direction of future work.

\section{Related Work}

The term "robotics" encompasses a variety of research subareas. One subarea is termed Social robotics and involves robots that engage in some form of social interaction with humans, through speech, gestures, or other means of communication [11]. Another subarea of robotics is Assistive robotics, which generally involves robots that aid people with disabilities, mainly for the physical and neurodevelopmental disabilities [12]. Socially Assistive Robotics (SAR) is a fast emerging field that develops from the intersection of these two and involves robots that are designed to help through advanced interaction driven by user needs (e.g. tutoring, physical therapy, daily life assistance, emotional expression) via multimodal interfaces (speech, gestures, and input devices) [13].

Because of the multifaceted expertise required to develop socially assistive systems, the field of SAR is naturally interdisciplinary and shares its challenges with robotics, physiology, psychology, and sociology, among other fields [14]. Also from a technological point of view, SAR merges various topics, such as mechatronics, artificial intelligence, and real-time control issues $[15,16]$. Indeed, SAR is currently tested in many fields of application with three primary roles: companion, coach, and play partner. For instance, these robots can assist in tasks ranging from coaching elderly patients in physical exercise to guiding visitors through museums $[17,18]$. In the field of education and care of children, SAR research mainly focuses on the therapeutic applications for children with developmental disabilities, like 
those affected by Autism Spectrum Disorder (ASD) [1923]. SAR was also successfully applied to personalise health education for children with diabetes [24-26] to assist either teachers in telling pre-recorded stories to preschool children [27] or parents in home education [28].

An important goal of SAR research is the production of permanent benefits that last after the robot is no longer physically present. It is fundamental to understand how interactions with robots and skills learned or rehearsed with an SAR system can be translated into real-world situations and to interactions with other people [29].

Recently, Benitti [30] reviewed the scientific literature on the use of robotics in schools and suggested that educational robotics can act as an element that enhances learning, if appropriately used. In particular, robotic assistants have the potential to overcome concerns about the physical effects of children's use of computer-based tools [31], because they encourage children to be mobile during a game (e.g. [32]). As an example, Fridin et al. [33] show that preschool children are more involved in a motor task when they interact with a robot rather than with a virtual agent. The robot can be a practical learning partner that motivates students in learning and elicits learning performance naturally [34].

Researchers have shown that a robot companion can help to improve engagement and elicit novel social behaviours in children with developmental disabilities, for instance with ASD [22] and Down-Syndrome (DS) [35]. Lehmann et al. [35] have also worked to combine behavioural intervention approaches with assistive technology, where established teaching methods are employed to elicit skills using a robot.

Robots create opportunities not only to learn from a nonthreatening, three-dimensional inanimate object, but also to learn through interaction with other human beings, thus encouraging autonomous social behaviour [27]. This has enabled robots to help with a variety of human-like functions, as well as to aid with the goal of improving social skills in individuals with disability [36].

The "WikiTherapist Project" is a very ambitious project which used the humanoid robot NAO [37]. This project aims to empower health researchers/practitioners with robot assistants or mediators in behavioural therapies for children with ASD. The goal of the project is to create a web-based community of therapists and robot practitioners who co-create robot behaviours and scenarios with different complexity. The expected result is a robotic environment that is easy to control by non-specialists, which is equipped with a library of behaviours, and scenarios that are found useful for behavioural training of people with ASD [38].
Also, a small humanoid robot is used in a pediatric hospital to implement cognitive-behavioural strategies (distraction and blowing) while children received a flu vaccination from practitioners [39]. A humanoid robot provides a highly engaging and entertaining distraction that is able to divert children's attention away from their worry of fear and pain of the vaccination. Thus, it was hypothesized that children who were distracted by a robot during their flu vaccination would experience lower levels of pain and distress than children without such distraction [40,41].

As we have seen, a robot will need to be programmed for different relevant tasks during treatment; for practical purposes, it would be ideal for therapists to have the ability to complete this programming themselves. Therefore, therapists and other users must be able to manage and understand how to use the robot for a variety of clinical needs. One potential concern is that the technological knowledge needed in order for mental healthcare consumers (e.g. patients, therapists) to use robots effectively may be beyond the existing skill sets of some users. The goals of the research endeavours are to create systems that can be easily and readily used for treatment applications to increase the acceptance of these instruments [29].

Research about robotics acceptance have often seen older people in assisted living scenarios as the main focus (e.g. [42-45]), but relatively few studies have been conducted with other participants such as children or their educators [46] and caregivers. In general robot acceptance by younger children is difficult to assess because it is not possible to reliably administer the common questionnaires, thus acceptance factors are indirectly derived observing the interaction [47]. School children's perceptions and evaluations of different robot designs were studied by Woods [48]. A large sample $(\mathrm{N}=159)$ of children evaluated 40 robot images and judged human-like robots as aggressive, but human-machine robots (i.e. with human-like features in addition to some machinelike traits) as friendly. This result on children's perceptions of the robots' behavioural intentions provided tentative empirical support for the Uncanny Valley, hypothesized by Mori [49]. The Uncanny Valley is a phenomenon in the field of aesthetics which affirms that when features look like, but not exactly, natural beings, this may generate a response of repulsion among some observers [50].

In a recent study for employees of a disability service organization, with an online-delivered exploration without a real robot, the most participants believed that robots cannot replace the human touch, human interaction, or emotional companionship, and that they cannot (should not) replace human workers in the disability setting. Also, many expressed concerns about safety, normality for disabled people, and artificial interactions [3]. 


\section{Material and Methods}

\subsection{Technology Acceptance Models and Hypotheses}

The first studies on technology acceptance ${ }^{1}$ modelling usually can be traced back to Davis with the Technology Acceptance Model (TAM) [51]. According to this model, which has been used for different types of technology, the user perception of utility and ease of use of a system determines the intention and afterwards the actual use of the system itself. Subsequently Venkatesk et al. [52] published an inventory of current models and factors and presented a model called Unified Theory of Acceptance and Use of Technology (UTAUT) in which all relevant factors were incorporated. An extensive literature review has been conducted by de Graaf et al. [53] and they provided an overview of variables influencing the acceptance of social robots categorized by utilitarian variables, hedonic variables, user characteristics, social normative beliefs and control beliefs. In this study, our objective was to evaluate the participant perception, attitude and intention of use a SAR platform in their profession. To this end we adopted the UTAUT model as proposed by Heerink et al. [54], which has been widely used for the evaluation of SAR platforms and has been found to be highly reliable in several previous studies (among others: [25,55-58]).

Heerink et al. [56] show the interrelations between constructs, which can be used as hypotheses when the model is used for a specific system. The hypotheses considered here are as follows:

H1-Intention to use is determined by (a) perceived usefulness, (b) perceived ease of use, (c) attitude, (d) perceived enjoyment, (e) social influence.

H3 - Perceived usefulness is influenced by (a) perceived ease of use (b) perceived adaptability and (c) anxiety.

H5-Perceived enjoyment is influenced by (a) social presence and (b) perceived sociability.

H6-Perceived sociability is influenced by trust.

H7-Social presence is influenced by perceived sociability.

In total, Heerink et al. [56] defined seven hypotheses when the entire model is used, but $\mathrm{H} 2$ and $\mathrm{H} 4$ cannot be applied in our study, because it was not possible to gather data on actual use of the system by the participants.

\subsubsection{Questionnaire}

The UTAUT model uses a structured questionnaire to measure the variables that can influence the acceptance of

1 With the term "user acceptance" we mean "the demonstrable willingness within a user group to employ technology for the tasks it is designed to support" [78]. technology. Each possible influence, named "construct", is represented by a few questions and the scores for the constructs can be mapped and interrelated. For our study, we used the questionnaire proposed in Heerink et al. [56], in which we replaced the references to the iCat with the general word "robot". The participants could indicate their level of agreement to 36 statements on a five-point Likert scale including verbal anchors: totally disagree (1)-disagree (2)neither agree nor disagree (3)-agree (4)-totally agree (5).

The original questionnaire was translated into Italian using a back-translation process employing a bilingual speaker. This procedure ensures that meaning and linguistic nuances are not lost and that the translated version remains as true to the original construct as possible. Prior to the main study, a pilot test $(\mathrm{N}=4)$ for the questionnaire was conducted to confirm the clarity of the wording of the questions and of the instructions. Comments collected during the administration of the pilot format were employed to create the final version of the questionnaire. Only question 6 was significantly modified from the original version of the questionnaire in order to make it more coherent with the two different groups of our sample: the word "life" was replaced by "job" or "future job", according to the group to be interviewed.

\subsection{Experimental Setup}

\subsubsection{The NAO Humanoid Robotic Platform}

The robotic platform used in our experiments is a NAO robot [59] that is $58 \mathrm{~cm}$ tall humanoid robot that looks like a toy. In the various studies that have used it, this robot was perceived by the participants as a smart, non-threatening educational tool [60] with whom children and the elderly can positively interact $[61,62]$. The robot speaks with a child's voice, expresses emotions (through verbal and non-verbal communication), and uses proper vocabulary. The NAO robot has 25 degrees of freedom, which allows it to perform a variety of movements. NAO has pioneered the use of robotic toys as therapeutic and educational aides and it is widely used in SAR (e.g. $[33,63,64])$, particularly in acceptance studies (e.g. $[53,65])$.

To program the NAO's behaviours we used Choreographe, a development environment provided by the robot manufacturer Aldebaran Robotics [66]. The interface is mainly drag and drop, and allows the programmer to create a sequenced combination of predefined or custom behaviour boxes to manipulate the NAO's joints or attributes, such as its voice or LED colours. Using Choreographe, we developed a set of pre-programmed behaviours to allow the robot to interact with the participants and we installed them on the NAO's memory. This way we were able to recall the behaviours during the experiments through a simple application for smartphones/tablets that lists all the available behaviours and 
allows sending text to speech. We retrieved some complex behaviour from the NAO database, like for instance dancing while playing music and an interactive game of picture recognition. Other custom behaviours were created combining simple actions with text to speech (e.g. waving the hand and say goodbye). All the behaviours originally created in other languages (e.g. the image recognition game) were translated into Italian. In the image recognition game, the robot was programmed to identify a printed image, among the ten stored in its memory (e.g. butterfly, tree, heart, and so on) and recognize if the one shown was correct or not. The recognition is communicated to the participant via speech and gestures. The images were selected to have the highest reliability of the recognition. Indeed, in our experiment the recognition rate was $100 \%$. Images can be rotated, but they must be fully in the robot field of vision to be recognized.

\subsubsection{Participants}

The entire research involved 80 participants, all of Italian nationality. All participants had no previous experience of interaction with social robotic platforms. Table 1 shows the descriptive statistics of the groups: 25 educations and rehabilitation care practitioners, all of them have obtained at least a university degree equivalent to a master, and 55 university students. Practitioners' professional experience ranges from 2 to 38 years, with an average of 17 years $(S D=11.2)$.

The practitioners group was composed of professionals with different specialization (neuro-psychiatrists, clinical psychologist, educators, and nurses) that work daily with people (children and elderly) with mental disabilities at the research and healthcare centre IRCCS Oasi Maria SS of Troina, located in the province of Enna (Italy). The students group was composed of two subgroups attending two different degree courses: the bachelor of Education sciences from the University of Enna Kore and the Master in Psychology (clinical rehabilitation track) from University of Catania. The Centre and the Universities are located in two neighbouring provinces of the same region (Sicily), from which the great majority of the students come from.

Table 1 Descriptive statistics of the sample

\begin{tabular}{|c|c|c|c|c|c|c|c|}
\hline \multirow[t]{2}{*}{ Group } & \multirow[t]{2}{*}{ Sub group } & \multicolumn{4}{|l|}{ Age } & \multicolumn{2}{|c|}{ Gender } \\
\hline & & Mean & Min & $\operatorname{Max}$ & SD & M & $\mathrm{F}$ \\
\hline \multicolumn{2}{|c|}{ Practitioners } & 41.8 & 26 & 63 & 11.6 & 5 & 20 \\
\hline \multirow[t]{3}{*}{ Students } & Educ & 22.8 & 20 & 37 & 5.1 & 6 & 12 \\
\hline & Psyc & 27.1 & 23 & 37 & 3.1 & 3 & 34 \\
\hline & All & 25.7 & 20 & 37 & 4.3 & 9 & 46 \\
\hline Total & & 30.7 & 20 & 63 & 10.5 & 14 & 66 \\
\hline
\end{tabular}

\subsubsection{Experimental Procedure}

The experiment was performed in rooms that had a good light and there was no background noise. Before the beginning of the experiment, the NAO robot was placed on a table to ensure good visibility for all participants. The total time was about thirty minutes and it was divided into two parts. In the first part, participants took part in an interactive demonstration of the NAO robot capabilities. In the second part, all participants anonymously filled the UTAUT questionnaire immediately after the live demonstration. A final discussion was held to allow participants to express their own thoughts and, thus, provide more information for the research. All participants of each group or sub-group attended the demonstration together.

At the beginning of the first part, the use of robots in the care of children and disabled was briefly presented. The presentation was carried out in two phases: first a familiar authority (the head of the department for practitioners, a teacher for the students) listed some of the possible uses and applications in the education and rehabilitation of children with developmental disabilities; then, the researcher explained the experiment and described the NAO robot functionalities linking them with the possible applications, using the same words for all groups. At the beginning of the demonstration, the robot welcomed the participants and began a dance to give the opportunity to see the harmony of its movements. Next, the robot proposed that the participants played an interactive game of image recognition. Sheets with printed images were placed on the table that a random volunteer selected and showed to the robot when asked (e.g. "I would like to see a butterfly"). In our experiments, the robot was able to recognize all the images presented, but some participants spent tens of seconds to find the correct position in the robot field of vision. The game was repeated several times to allow many participants to actually interact with the robot. After the game, the robot was placed down on the floor to demonstrate its walking capability for five minutes. In this phase, participants could verbally indicate the direction (e.g. they can say "forward", "backward", and "turn" "left" or "right") that the robot should go. Finally, the robot thanked the participants for attending and for their participation. The final messages were customized taking into account the different groups of participants. This was to make the interaction with the robot more personalized and friendly.

The robot used informal language and expressed enjoyment through body motion and gestures. Not all participants interacted directly with the robot, but all the spectators were following the demonstration and interaction sessions with great attention and we noted the same emotional involvement as they were playing with the robot in first person. In fact, the Perceived Enjoyment (PENJ) construct was posi- 

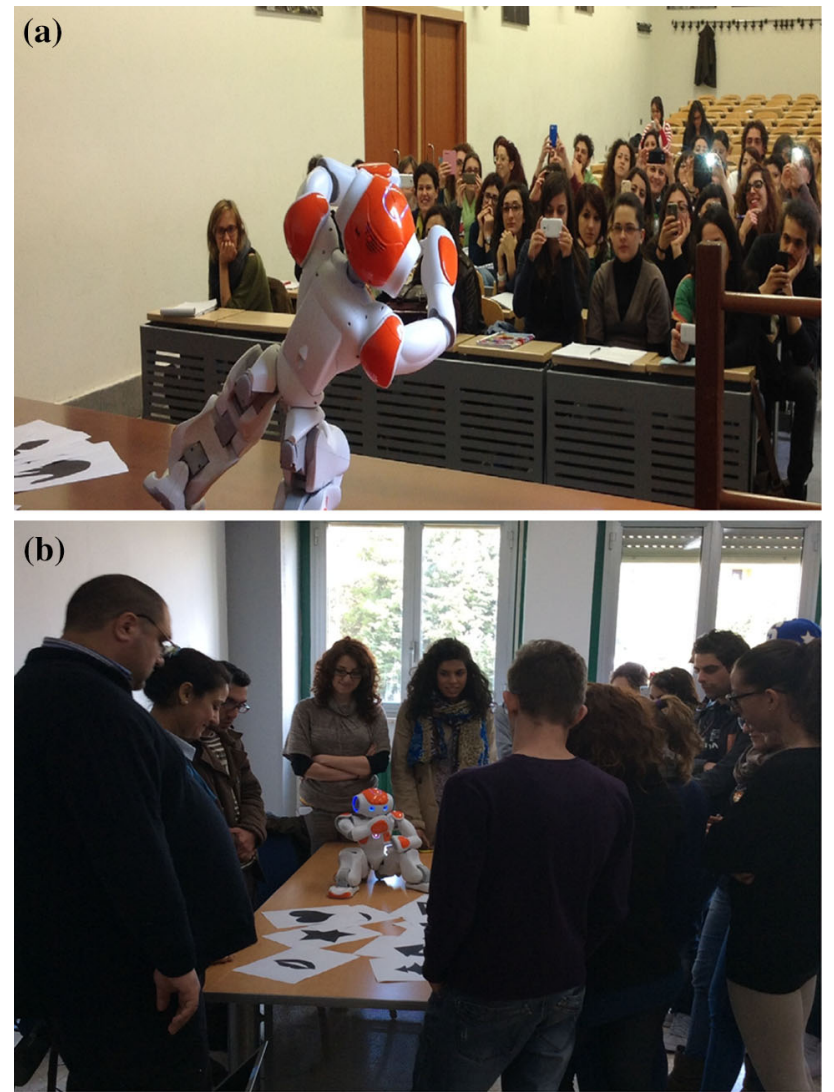

Fig. 1 a Students watching the movements of the NAO robot; b students playing the picture recognition game

tively scored by the greater majority of the participants. This behaviour was also noted by Fridin et al. [33]. In all groups, almost all participants took a picture or a video of the robot with their own mobile phone/tablet (see Fig. 1a). Figure 1b shows the students playing the image recognition game.

The same experimental procedure was used for both students and practitioners.

\subsection{Data Analyses}

To process the results we employed two sets of analyses. The first set aims to validate the questionnaire and to test the UTAUT model hypotheses in the context of education and care of children with developmental disabilities. The other set of analyses aims to compare the groups and perform qualitative analyses on the acceptance by the practitioners and the students.

The UTAUT data were processed as follows:

- Reliability of the questionnaire was established by calculating Cronbach's alpha for the items of each construct. We consider an acceptable alpha value if it is greater than .600, while a solid construct would have an alpha of at least .700 (for details see [67]).
-When a construct consisted of more than two statements, we tested what the alpha score would be if one or more questions are omitted. If a more solid argument is obtained the statement is removed from the calculation of the construct score.

- The scores for each construct were calculated by averaging the scores on the items.

- Hypotheses were tested with correlations (exploratory analysis) and linear regression analysis (confirmative). For each hypothesis, we established the $\mathrm{R}^{2}$ value, which can be used as an indication of the predictive strength of the model.

- Correlation scores of each construct with age of participants were calculated to evaluate the possible impact of this variable on the results. Age was found to significantly $(\mathrm{p}<.05)$ correlate to the Intention to Use (ITU) by Heerink [68].

Group comparisons and qualitative analyses on the acceptance were performed with the following measures:

- A positive perception (POS) of a participant is assumed when the construct score is greater than 3, while a negative perception (NEG) is when average score is lower than 3. Otherwise, the perception is considered neutral.

- Basic descriptive statistics (minimum and maximum scores, mean and variance) and of positive and negative perception (percentage).

- The Mann-Whitney U (a.k.a. Mann-Whitney-Wilcoxon) test was preferred to test the independence of the samples, because it's more general applicability and, thus, reliability when samples have different distribution, size (i.e. number of subjects) and variance [69].

All statistics were calculated using SPSS 21 software.

\section{Results}

Table 2 shows the constructs included in the questionnaire and the Cronbach's alpha for each of them. Note that, in this article, questionnaire statements are referred to using the construct code to which they belong, and the number corresponding to its position in the list. As an example ITU10 identifies the 10th statement that belongs to the construct ITU.

According to Alpha values, the reliability of the majority of constructs is solid. As reported in the notes (Table 2) we removed question 1, 2 and 14 to improve the reliability of the constructs ANX and PAD. Note that SI is formed by only two items so no improvement was possible, but we consider that alpha score of .629 as acceptable. The only construct that we found unreliable is FC, which is lower than .400 . 
Table 2 Constructs Cronbach's alpha

\begin{tabular}{llll}
\hline Code & Construct & Num. Items & Cronbach's alpha \\
\hline ANX & Anxiety & 4 & $.872^{*}$ \\
ATT & Attitude & 3 & .762 \\
FC & Facilitating conditions & 2 & $.357^{* *}$ \\
ITU & Intention to use & 3 & .874 \\
PAD & Perceived adaptability & 3 & $.741^{* * *}$ \\
PENJ & Perceived enjoyment & 5 & .855 \\
PS & Perceived sociability & 4 & .812 \\
PU & Perceived usefulness & 3 & .831 \\
SI & Social influence & 2 & .629 \\
SP & Social presence & 5 & .729 \\
TR & Trust & 2 & $.933 \mathrm{~s}$ \\
\hline
\end{tabular}

*Alpha is improved by removing questions 1 and 2 . The score with all items was .606

**Given the low score, this construct is removed from the other analyses. Questions are evaluated individually

**Alpha is improved by removing question 14 . The score with all items was .621

This can be due to the limited experience in using the robot and programming its behaviours. A similar result for FC was founded by Fridin et al. [57]. For this reason, questions FC-8 and FC-9 are evaluated individually.

The correlation matrix of constructs is reported in Table 3 . Correlations clearly show that the constructs are related each other, only in a few cases there is not a significant correlation, while in many others we report a strong coefficient. Using the correlation scores to explore the hypotheses, we can conclude that they could be confirmed.

The correlation with age is low and not statistically significant ( $p>.05$ ) for the majority of constructs, including the ITU, but with the exception of PENJ and PS that seems to be negatively affected by age. Professional experience is highly $(\mathrm{r}=.939, \mathrm{p}<.0001)$ correlated with age of participants and it correlates with PENJ and PS too. As regards questions FC-8
Table 4 Linear regression analyses (separately for each hypothesis$\mathrm{N}=80$ )

\begin{tabular}{llllll}
\hline Hypothesis & $\begin{array}{l}\text { Independent } \\
\text { variables }\end{array}$ & $\begin{array}{l}\text { Dependent } \\
\text { variable }\end{array}$ & $\mathrm{R}^{2}$ & Beta & $\mathrm{t}$ \\
\hline H1 & ATT & ITU & .544 & .111 & .903 \\
& PENJ & & & .169 & 1.454 \\
& PU & & & .505 & $\mathbf{. 1 5 4 *}$ \\
& SI & & & .051 & .547 \\
H3 & PAD & PU & .444 & .217 & $\mathbf{2 . 5 2 1 *}$ \\
& ANX & & & .599 & $\mathbf{6 . 9 7 6}$ \\
H5 & SP & PENJ & .566 & .762 & $\mathbf{8 . 0 3 6}$ \\
& PS & & & -.016 & -.164 \\
H6 & TR & PS & .148 & .384 & $\mathbf{3 . 6 7 7 *}$ \\
H7 & PS & SP & .374 & .612 & $\mathbf{6 . 8 2 6 *}$ \\
\hline
\end{tabular}

Significance levels: $* \mathrm{p}<.05 ; * * \mathrm{p}<.01$

and FC-9, considered separately because the unreliability of the FC construct, they don't have any statistically significant correlation with other constructs

Table 4 reports the scores of the regression analyses of the UTAUT hypotheses for the entire sample. All hypotheses are confirmed at least partially.

Table 5 presents the descriptive statistics of the constructs: the minimum ( $\min$ ) and maximum (max) average scores and the percentage of positive (POS) and negative (NEG) perception of the participants.

The main difference between the two groups is on the intention to use (ITU), because it determines the actual use (hypothesis H1). We could say that practitioners were not convinced to actually use the robot in their job, indeed the average is below 3 with a higher number of negative than positive statements: 36 versus $24 \%$. We noted that practitioners' highest average score for ITU is 4 , i.e. no one in this group scored a 5. Meanwhile, students show more willingness to use the robot (i.e. $50 \%$ of ITU statements are above 3 ).
Table 3 Triangular correlation matrix among constructs and age for all participants $(\mathrm{N}=80)$

\begin{tabular}{|c|c|c|c|c|c|c|c|c|c|c|}
\hline & AGE & ANX & ATT & ITU & PAD & PENJ & PS & PU & SI & SP \\
\hline ANX & -.039 & 1 & & & & & & & & \\
\hline ATT & -.009 & $.352 * *$ & 1 & & & & & & & \\
\hline ITU & -.117 & $.282 *$ & $.585 * *$ & 1 & & & & & & \\
\hline PAD & -.079 & .147 & $.587 * *$ & $.667 * *$ & 1 & & & & & \\
\hline PENJ & $-.278^{*}$ & $.327 * *$ & $.700 * *$ & $.584 * *$ & $.598 * *$ & 1 & & & & \\
\hline PS & $-.336^{* *}$ & .209 & $.486 * *$ & $.467 * *$ & $.526 * *$ & $.753 * *$ & 1 & & & \\
\hline PU & -.117 & $.304 * *$ & $.676 * *$ & $.712 * *$ & $.631 * *$ & $.628 * *$ & $.545^{* *}$ & 1 & & \\
\hline SI & -.115 & $.224 *$ & $.290 * *$ & $.414 * *$ & $.294 * *$ & $.398 * *$ & $.352 * *$ & $.523 * *$ & 1 & \\
\hline SP & -.211 & .167 & $.231 *$ & $.321 * *$ & .212 & $.450 * *$ & $.612 * *$ & $.336 * *$ & .178 & 1 \\
\hline TR & -.041 & -.026 & .182 & .210 & $.231 *$ & $.228 *$ & $.384 * *$ & $.243 *$ & .180 & $.428 * *$ \\
\hline
\end{tabular}

Significant values are in bold

Correlation is significant: $*$ at the .05 level; $* *$ at the .01 level 
Table 5 Comparison between practitioners and students: constructs analysis

\begin{tabular}{|c|c|c|c|c|c|c|c|c|c|c|c|c|c|}
\hline \multirow[t]{2}{*}{ Construct } & \multicolumn{6}{|c|}{ Practitioners } & \multicolumn{6}{|c|}{ Students } & \multirow{2}{*}{$\begin{array}{l}\text { Mean } \\
\text { Difference }\end{array}$} \\
\hline & Mean & Min & $\operatorname{Max}$ & $\mathrm{SD}$ & POS $(\%)$ & NEG $(\%)$ & Mean & Min & Max & SD & POS (\%) & NEG $(\%)$ & \\
\hline ANX & 4.24 & 2.50 & 5.00 & 0.90 & 80 & 8 & 4.44 & 2.00 & 5.00 & 0.84 & 87 & 7 & -0.20 \\
\hline ATT & 3.45 & 1.00 & 5.00 & 0.98 & 68 & 12 & 3.64 & 2.00 & 5.00 & 0.66 & 78 & 11 & -0.19 \\
\hline ITU & 2.75 & 1.00 & 4.00 & 0.83 & 24 & 36 & 3.18 & 1.00 & 5.00 & 0.82 & 51 & 22 & $-0.44^{*}$ \\
\hline PAD & 3.14 & 1.00 & 4.50 & 0.84 & 52 & 28 & 3.38 & 1.50 & 5.00 & 0.76 & 65 & 16 & -0.24 \\
\hline PENJ & 3.46 & 1.40 & 4.80 & 0.82 & 68 & 16 & 4.08 & 2.20 & 5.00 & 0.65 & 95 & 4 & $-0.62^{* *}$ \\
\hline PS & 2.45 & 1.00 & 4.00 & 0.78 & 16 & 72 & 3.20 & 1.50 & 5.00 & 0.82 & 51 & 31 & $-0.75^{* *}$ \\
\hline PU & 3.07 & 1.00 & 5.00 & 0.84 & 48 & 20 & 3.38 & 1.33 & 5.00 & 0.79 & 64 & 18 & -0.31 \\
\hline SI & 2.94 & 1.00 & 4.00 & 0.74 & 32 & 24 & 3.30 & 1.00 & 5.00 & 0.73 & 53 & 20 & -0.36 \\
\hline SP & 2.01 & 1.00 & 3.60 & 0.76 & 8 & 80 & 2.51 & 1.00 & 5.00 & 0.89 & 24 & 73 & $-0.50^{*}$ \\
\hline TR & 2.12 & 1.00 & 5.00 & 1.27 & 24 & 64 & 2.06 & 1.00 & 5.00 & 1.02 & 7 & 73 & 0.06 \\
\hline
\end{tabular}

Highest percentages and significant differences are in bold

Mann-Whitney U significance levels: * $\mathrm{p}<0.05 ; * * \mathrm{p}<0.01$

Table 6 Comparison between practitioners and students: single answers analysis

\begin{tabular}{|c|c|c|c|c|c|c|c|c|c|c|c|c|c|}
\hline \multirow[t]{2}{*}{ Question } & \multicolumn{6}{|c|}{ Practitioners } & \multicolumn{6}{|c|}{ Students } & \multirow{2}{*}{$\begin{array}{l}\text { Mean } \\
\text { Difference }\end{array}$} \\
\hline & Mean & Min & Max & SD & $\operatorname{POS}(\%)$ & NEG $(\%)$ & Mean & Min & Max & $\mathrm{SD}$ & $\operatorname{POS}(\%)$ & NEG $(\%)$ & \\
\hline \multicolumn{14}{|c|}{ Questions removed from constructs after Cronbach's alpha analysis } \\
\hline ANX-1 & 3.48 & 1 & 5 & 1.41 & 56 & 28 & 3.53 & 2 & 5 & 0.97 & 49 & 16 & -0.05 \\
\hline ANX-2 & 3.84 & 2 & 5 & 1.07 & 60 & 12 & 3.09 & 1 & 5 & 1.13 & 36 & 40 & $\mathbf{+ 0 . 7 5} * *$ \\
\hline FC-8 & 2.52 & 1 & 5 & 1.23 & 24 & 48 & 2.60 & 1 & 5 & 1.01 & 20 & 53 & -0.08 \\
\hline FC-9 & 2.16 & 1 & 5 & 1.43 & 20 & 68 & 2.27 & 1 & 5 & 1.15 & 16 & 67 & -0.11 \\
\hline PAD-14 & 3.64 & 1 & 5 & 0.81 & 64 & 4 & 3.51 & 1 & 5 & 0.92 & 60 & 16 & +0.13 \\
\hline \multicolumn{14}{|c|}{ Questions with significant differences between groups } \\
\hline ATT-5 & 3.52 & 1 & 5 & 1.08 & 56 & 12 & 4.11 & 2 & 5 & 0.81 & 84 & 5 & $-0.59^{*}$ \\
\hline ATT-6 & 3.56 & 1 & 5 & 1.12 & 56 & 12 & 3.02 & 1 & 5 & 0.93 & 25 & 22 & $+0.54 *$ \\
\hline ITU-12 & 2.68 & 1 & 4 & 0.90 & 16 & 36 & 3.33 & 1 & 5 & 0.96 & 45 & 13 & $-0.65^{* *}$ \\
\hline PENJ-19 & 3.40 & 1 & 5 & 1.04 & 48 & 12 & 4.27 & 2 & 5 & 0.83 & 84 & 4 & $-0.87^{* *}$ \\
\hline PS-21 & 2.36 & 1 & 4 & 1.08 & 16 & 52 & 3.36 & 1 & 5 & 1.01 & 47 & 16 & $-1.00^{* *}$ \\
\hline PS-22 & 2.92 & 1 & 4 & 1.00 & 32 & 28 & 3.87 & 2 & 5 & 0.75 & 76 & 5 & $-0.95^{* *}$ \\
\hline SI-29 & 2.79 & 1 & 4 & 0.72 & 8 & 20 & 3.30 & 1 & 5 & 0.84 & 40 & 11 & $-0.51^{* *}$ \\
\hline SP-31 & 1.96 & 1 & 4 & 1.10 & 8 & 60 & 2.91 & 1 & 5 & 1.34 & 36 & 42 & $-0.95^{* *}$ \\
\hline
\end{tabular}

Highest percentages and significant differences are in bold

Mann-Whitney U test significance level: $* \mathrm{p}<0.05 ; * * \mathrm{p}<.01$

Table 6 reports the questions removed from the constructs after the Cronbach's alpha analysis, and those included in the constructs that received the most significantly different answers, in terms of the mean difference between the two groups, statistical significance $(\mathrm{p}<.01)$ and impact on the constructs. We analysed the single questions removed from the constructs to avoid losing some information because of the removal. In fact, none had a statistically significant difference, with the only exception of ANX-2. In comparison, practitioners are more self-confident because the majority of them expressed low (4) or no anxiety (5) to damage the robot (ANX-2) and they think that the robot would make their job more interesting (ATT-6). On the other hand, students strongly support the idea to use the robot (ATT-5), while they have no particular opinion on ATT-6, probably because they haven't a concrete work experience yet. Students consider the interaction with the robot pleasant (PS-21/22), indeed practically all of them really enjoyed the demonstration, and they think that the robot is fascinating (PENJ-19). Vice versa the majority of practitioners do not consider the robot as a pleasant conversational partner (PS-21). The factor that has a more negative influence on the practitioner's perception of robot sociability is that they do not think it is actually looking at them (SP-31). Students have an almost neutral perception 
on SP-31, which is significantly higher than the practitioners. A total of $40 \%$ of students perceive that there is a social influence toward using the robot and that they will give a good impression if they should use the robot (SI-29), while practitioners are generally neutral $(72 \%)$ or slightly negative (20\%) about this statement. As a final consideration, $45 \%$ of students say that they are planning to use the robot in their job while only $13 \%$ are not (ITU-12), while for practitioners percentages are reversed and the maximum score given to this question was 4.

Finally, comparing the student subgroups we report that there are no statistically significant $(\mathrm{p}>0.1)$ differences between the average scores of the constructs according to the Mann-Whitney U test analysis.

\section{Discussion}

Results obtained in our study confirmed the reliability of the UTAUT model and questionnaire in the evaluation of the acceptance and the intention to use a robotic platform by professionals involved in the education and care of children with developmental disabilities.

The correlation and regression analyses confirmed, fully or partially, the hypotheses of the UTAUT model. In particular, the analysis shows that Intention to use (ITU) is influenced by the Perceived usefulness (PU) only. In other words, the desire to use the robot (or not) is mainly predicted by the perception that it will enhance and facilitate the educational and therapeutic process. This is similar to results found by previous studies $[57,70]$ but only partially confirms the hypothesis $\mathrm{H} 1$ of Heerink et al. [56].

Our analysis supports hypothesis H3, because Perceived usefulness (PU) is related to anxiety and perceived adaptability. Indeed, the robot adaptability to different contexts is one of the questions that were asked by some of the participants after the demonstration. Hypothesis H5 is partially confirmed, because Perceived enjoyment (PENJ) is related only to the Social presence (SP), but not to the Perceived sociability (PS). However, given that hypothesis $\mathrm{H} 7$ is confirmed and SP is determined by PS, we can conclude that Perceived enjoyment (PENJ) is indirectly determined by Perceived sociability (PS). Finally, hypothesis H6 is confirmed as Perceived sociability (PS) is determined by Trust (TR). In addition, we found also that perceived enjoyment and sociability correlate with age, that was observed in previous work [53].

The differences in the relationship between the constructs with respect to the hypotheses formulated in Heerink et al. [56] can be explained by the fact that they were focused on the elderly and their acceptance of a small robot as a companion, while we are investigating its use as an instrument for professional practice with intellectually disabled.
The Facilitating Conditions (FC) result is clearly limited by the short time spent with the robot. The answers are generally negative and reflect the missing knowledge of the languages and tools to program the robot behaviour. In this case, we can hypothesize the participant derived their answers from previous experience with the technology [71].

When we compare the two groups in our sample, we see a more positive Intention to use (ITU) the robot by the students, while this variable is negative for the practitioners. In particular, the majority of the practitioners said that they are not planning to use the robot in the near future.

The main factor of this difference is obviously the professional experience, which has been found to influence the teacher intention to use the technology in the classroom [72]. In our sample, professional experience is highly related to age that we found to affect the perceived sociability (PS). Indeed, practitioners considered the experience with the robot less pleasant and, thus, this influenced negatively their acceptance and intention to use the robot [73]. Vice versa, students enjoyed the demonstration and consider the robot pleasant. Moreover, practitioners have a clearer view than students of the educational and therapeutic tools available and their effectiveness. They can easily identify the current technology difficulties and limitations, especially with higher levels of intellectual disabilities and this negatively affects the perceived usefulness. On the other hand, lack of experience leads the students to accept without questioning what they are told by the researchers, i.e. that the robot is a good idea (Attitude ATT-5), it is useful for their future work (Perceived usefulness-PU) and these considerations influences the Intention to use (ITU) the robot.

We see that students are neutral in ATT-6, while highly positive in Attitude ATT-5, this is probably because the question was directly asking them to predict the impact of the robot on their future work, so they tend to be neutral.

It should be pointed out that also majority of the practitioners agree that the robot is a good idea, but this does not determine the Intention to use (ITU), because, as our analysis highlight, the only factor that determines the Intention to use (ITU) is the Perceived usefulness (PU), which is negative for them.

As a confirmation of the relation between constructs, we underline that average score differences are reflected in the single questions of related constructs regardless of the positive or negative perception expressed by the majority of the group participants. For instance, we recall that SP-31 can determine PS-21/22 according to hypothesis H7, while PS21/22 determines Perceived enjoyment (PENJ) according to hypothesis H5. We see that students scored 0.95 more than practitioners in SP-31, in which we see a negative perception by both groups, then PS-21/22 student average score is respectively 1.00 and 0.95 higher, in which the majority of students are positive, while practitioners are negative, and, 
finally, student average scores of PENJ-19 are 0.87 higher than practitioners and the majority of both groups have a positive perception.

A factor interrelated with the professional experience is the Social influence (SI). The comparison shows that there is a significant difference in the perception of public recognition achievable by using the robot (question SI-29). This can be explained by the fact that many students perceived the use of the robot as enhancing their self-image or social status in the opinion of significant others, which, in turn, could have consequences for the user's acceptance of that innovation $[74,75]$.

Finally, during the open discussion after filling the questionnaire, practitioners expressed concern about the cost of the robot purchase. Similarly, Mutlu and Forlizzi [76] found that hospital workers have less willingness to use a robot if perceived benefits are outweighed by the costs, in terms of economical expense and workload for workers.

\section{Conclusions}

In this paper we reported the results of a study on acceptance of robots in the education and care of children by a group of 25 practitioners, specialized in developmental disabilities, and 55 students in psychology and educational disciplines. The study confirmed the reliability of the UTAUT model and its applicability in the context of education and care of children.

All participants showed a global positive attitude toward the use of the robot. The comparison between the two groups highlights the prevailing scepticism of the practitioners, while students show an overall positive perception and significantly higher willingness to use the robot. The result is due not only to differences in age (significant only for few variables), but to the professional experience of the practitioners that allows them to identify practical issues that could be encountered in the use of a robot with children affected by severe intellectual disabilities. We hypothesize that practitioners currently perceive the SAR as just an expensive and limited tool, which may provide a real advancement over other established techniques only if more synergistically integrated with standard protocols, in a way that benefits can outweigh the costs. To this end more evidence from the scientific research and integration with therapeutic protocols are needed to overcome the scepticism and draw firm conclusions about the clinical utility of robots [21].

The study is limited by the short amount of time that the participants spent interacting with the robot; they could be influenced by the limited previous knowledge of the specific technology [71]. On the other hand, in this work we were not testing a specific platform or the actual use of the robot, but the focus was on their general perception of SAR as a tool for education and care of children with developmental disabilities. Another limitation of the study is that possible applications and different platforms were only described and linked with the functionalities shown in the demonstration. Furthermore, the live experience with only one robotic platform could have biased the participant's perception. In future work, we will investigate the acceptance and usability of practitioners showing the real use of different robots as a therapeutic tool, e.g. with videotaped demonstrations.

We recall that people with different nationalities tend to rate differently their experiences with robots on usefulness, enjoyment, sociability, anthropomorphism, and perceived behavioural control [77], because each culture possesses its own level of exposure to robots through either media or personal experiences [9]. For this reason, another direction for future studies could be to extend the sample with students and practitioners of different nationalities, in order to assess the impact of culture on the results.

Open Access This article is distributed under the terms of the Creative Commons Attribution 4.0 International License (http://creativecomm ons.org/licenses/by/4.0/), which permits unrestricted use, distribution, and reproduction in any medium, provided you give appropriate credit to the original author(s) and the source, provide a link to the Creative Commons license, and indicate if changes were made.

\section{References}

1. TNS Opinion \& Social, Public Attitudes towards Robots, 2012

2. European Commission (2012) Eurobarometer Special 382: Public Attitudes towards Robots, no. May. Brussels, Belgium

3. Wolbring G, Yumakulov S (2014) Social Robots: views of Staff of a Disability Service Organization. Int J Soc Robot 6(March):457468

4. Moon AJ, Danielson P, van der Loos HFM (2012) Survey-based discussions on morally contentious applications of interactive robotics. Int J Soc Robot 4:77-96

5. Sparrow R, Sparrow L (2006) In the hands of machines? The future of aged care. Minds Mach 16:141-161

6. Coeckelbergh M, Pop C, Simut R, Peca A, Pintea S, Pintea S, David D, Vanderborght B (2015) A Survey of Expectations About the Role of Robots in Robot-Assisted Therapy for children with ASD: ethical acceptability, ttust, sociability, appearance, and attachment. Sci Eng Ethics 22:1-19

7. Ray C, Mondada F, Siegwart R (2008) What do people expect from robots? In: 2008 IEEE/RSJ international conference on intelligent robots and systems IROS, pp 3816-3821

8. Dautenhahn K, Woods S, Kaouri C, Walters ML, Koay KL, Werry I (2005) What is a robot companion-friend, assistant or butler? In: 2005 IEEE/RSJ international conference on intelligent robots and Systems IROS, pp 1488-1493

9. Broadbent E, Stafford R, MacDonald B (2009) Acceptance of healthcare robots for the older population: review and future directions. Int J Soc Robot 1:319-330

10. Kanda T, Miyashita T, Osada T, Haikawa Y, Ishiguro H (2008) Analysis of humanoid appearances in human-robot interaction. Robot IEEE Trans 24(3):725-735

11. Fong T, Nourbakhsh I, Dautenhahn K (2003) A survey of socially interactive robots. Robot Auton Syst 42:143-166 
12. Miller DP (1998) Assistive robotics: an overview, in assistive technology and artificial intelligence. Springer, New York, pp 126-136

13. Feil-Seifer D, Mataric MJ (2005) Defining socially assistive robotics, Rehabilitation robotics, ICORR 2005. In: 9th international conference, pp 465-468

14. Tapus A, Mataric MJ, Scasselati B (2007) ocially assistive robotics [grand challenges of robotics]. IEEE Robot Autom Mag 14:35-42

15. Feil-Seifer D, Matarić MJ (2011) Automated detection and classification of positive vs. negative robot interactions with children with autism using distance-based features. In: Proceedings of the 6th international conference on human-robot interaction, pp 323-330

16. McColl D, Nejat G (2014) Recognizing emotional body language displayed by a human-like social robot. Int J Soc Robot 6(2):261280

17. Fasola J, Matarić MJ (2013) A socially assistive robot exercise coach for the elderly. J Human-Robot Interact 2(2):3-32

18. Yousuf MA, Kobayashi Y, Kuno Y, Yamazaki K, Yamazaki A (2012) Establishment of spatial formation by a mobile guide robot. In: 7th ACM/IEEE international conference on human-robot interaction (HRI), pp 281-282

19. Feil-Seifer D, Matarić MJ (2009) Toward socially assistive robotics for augmenting interventions for children with autism spectrum disorders, in springer tracts. Adv Robot 54:201-210

20. Robins B, Dautenhahn K, Te Boekhorst R, Billard A (2005) Robotic assistants in therapy and education of children with autism: can a small humanoid robot help encourage social interaction skills? Univers Access Inf Soc 4(2):105-120

21. Diehl JJ, Schmitt LM, Villano M, Crowell CR (2012) The clinical use of robots for individuals with autism spectrum disorders: a critical review. Res Autism Spectr Disord 6(1):249-262

22. Scassellati B, Admoni H, Matarić M (2012) Robots for use in autism research. Annu Rev Biomed Eng 14:275-294

23. Conti D, Di Nuovo S, Trubia G, Buono S, Di Nuovo A (2015) Use of robotics to stimulate imitation in children with autism spectrum disorder: a pilot study in a clinical setting. In: IEEE RO-MAN, pp $1-6$

24. Blanson OA, Bierman BPB, Janssen J, Neerincx MA, Looije R, Van der Bosch H, Van der Giessen JAM (2013) Using a robot to personalise health education for children with diabetes type 1: a pilot study. Patient Educ Couns 92(2):174-181

25. Looije R, Cnossen F, Neerincx MA (2006) Incorporating guidelines for health assistance into a socially intelligent robot. In: Proceedings-IEEE international workshop on robot and human interactive communication, pp 515-520

26. Belpaeme T, Baxter PE, Read R, Wood R, Cuayáhuitl H, Kiefer B, Racioppa S, Kruijff-Korbayová I, Athanasopoulos G, Enescu V (2012) Multimodal child-robot interaction: building social bonds. J Human-Robot Interact 1(2):33-53

27. Fridin M (2014) Storytelling by a kindergarten social assistive robot: a tool for constructive learning in preschool education. Comput Educ 70:53-64

28. Han JHJ, Jo MJM, Park SPS, Kim SKS (2005) The educational use of home robots for children, Rom. In: IEEE international workshop on robot and human interactive communication

29. Rabbitt SM, Kazdin AE, Scassellati B (2014) Integrating socially assistive robotics into mental healthcare interventions: applications and recommendations for expanded use. Clin Psychol Rev 35:3546

30. Benitti FBV (2012) Exploring the educational potential of robotics in schools: a systematic review. Comput Educ 58(3):978-988

31. Dockrell S, Earle D, Galvin R (2010) Computer-related posture and discomfort in primary school children: the effects of a school-based ergonomic intervention. Comput Educ 55:276-284

32. Tanaka F, Movellan JR, Fortenberry B, Aisaka K (2006) Daily HRI evaluation at a classroom environment: reports from dance inter- action experiments. In: Proceedings of the 1 st annual conference on human-robot interaction HRI, pp 3-9

33. Fridin M, Belokopytov M (2014) Embodied robot versus virtual agent: involvement of preschool children in motor task performance. Int J Hum Comput Interact 30(6):459-469

34. Chang C-W, Lee J-H, Wang C-Y, Chen G-D (2010) Improving the authentic learning experience by integrating robots into the mixedreality environment. Comput Educ 55(4):1572-1578

35. Lehmann H, Iacono I, Dautenhahn K, Marti P, Robins B (2014) Robot companions for children with down syndrome: a case study. Interact Stud 15(1):99-112

36. Houwen S, van der Putten A, Vlaskamp C (2014) A systematic review of the effects of motor interventions to improve motor, cognitive, and/or social functioning in people with severe or profound intellectual disabilities. Res Dev Disabil 35(9):2093-2116

37. Gillesen J, Boere S, Barakova E (2010) WikiTherapist. In: Proceedings of the 28th annual European conference on cognitive ergonomics-ECCE' 10

38. Thill S, Pop CA, Belpaeme T, Ziemke T, Vanderborght B (2012) Robot-assisted therapy for autism spectrum disorders with (partially) autonomous control: challenges and outlook. Paladyn 3(4):209-217

39. Beran TN, Ramirez-Serrano A, Vanderkooi OG, Kuhn S (2013) Reducing children's pain and distress towards flu vaccinations: a novel and effective application of humanoid robotics. Vaccine 31(25):2772-2777

40. Taddio A, Appleton M, Bortolussi R, Chambers C, Dubey V, Halperin S, Hanrahan A, Ipp M, Lockett D, MacDonald N, Midmer D, Mousmanis P, Palda V, Pielak K, Riddell RP, Rieder M, Scott J, Shah V (2010) Reducing the pain of childhood vaccination: an evidence-based clinical practice guideline (summary). CMAJ 182:1989-1995

41. Uman LS, Chambers CT, McGrath PJ, Kisely S (2008) A systematic review of randomized controlled trials examining psychological interventions for needle-related procedural pain and distress in children and adolescents: an abbreviated cochrane review. J Pediatr Psychol 33:842-854

42. Chen K, Chan AHS (2011) A review of technology acceptance by older adults. Gerontechnology 10(1):1-12

43. Broekens J, Heerink M, Rosendal H (2009) Assistive social robots in elderly care: a review. Gerontechnology 8(2):94-103

44. Smarr C-A, Prakash A, Beer JM, Mitzner TL, Kemp CC, Rogers WA (2012) Older adults' preferences for and acceptance of robot assistance for everyday living tasks. Proc Hum Factors Ergon Soc Annu Meet 56(1): 153-157

45. Klamer T, Ben Allouch S (2010) Acceptance and use of a social robot by elderly users in a domestic environment. In: Pervasive computing technologies for healthcare (PervasiveHealth), 2010 IEEE 4th international conference, $\mathrm{pp}$ 1-8

46. Baroni I, Nalin M, Baxter P, Pozzi C, Oleari E, Sanna A, Belpaeme $\mathrm{T}$ (2014) What a robotic companion could do for a diabetic child. In: Robot and human interactive communication. 2014 RO-MAN: the 23rd IEEE international symposium, pp 936-941

47. Salter T, Werry I, Michaud F (2008) Going into the wild in childrobot interaction studies: issues in social robotic development. Intell Serv Robot 1(2):93-108

48. Woods S (2006) Exploring the design space of robots: children's perspectives. Interact comput 18:1390-1418

49. Mori M, MacDorman KF, Kageki N (2012) The uncanny valley [from the field]. Robot Autom Mag IEEE 19(2):98-100

50. MacDorman KF, Ishiguro H (2006) The uncanny advantage of using androids in cognitive and social science research. Interact Stud 7(3):297-337

51. Davis F (1989) Perceived usefulness, perceived ease of use, and user acceptance of information technology. MIS Quat 13:319-340 
52. Venkatesh V, Morris M, Davis G, Davis F (2003) User acceptance of information technology: toward a unified view. MIS Quat 27:425478

53. De Graaf MMA, Ben Allouch S (2013) Exploring influencing variables for the acceptance of social robots. Robot Auton Syst 61:1476-1486

54. Heerink M, Kröse B, Evers V, Wielinga B (2009) Measuring acceptance of an assistive social robot: A suggested toolkit. In: Proceedings-IEEE international workshop on robot and human interactive communication, pp 528-533

55. De Ruyter B, Saini P, Markopoulos P, Van Breemen A (2005) Assessing the effects of building social intelligence in a robotic interface for the home. Interact Comput 17:522-541

56. Heerink M, Kröse B, Evers V, Wielinga B (2010) Assessing acceptance of assistive social agent technology by older adults: the almere model. Int J Soc Robot 2:361-375

57. Fridin M, Belokopytov M (2014) Acceptance of socially assistive humanoid robot by preschool and elementary school teachers. Comput Human Behav 33:23-31

58. Conti D, Cattani A, Di Nuovo S, Di Nuovo A (2015) A crosscultural study of acceptance and use of robotics by future psychology practitioners. In: 24th IEEE international symposium on robot and human interactive communication, RO-MAN, pp 555-560

59. Gouaillier D, Hugel V, Blazevic P, Kilner C, Monceaux J, Lafourcade P, Marnier B, Serre J, Maisonnier B (2009) Mechatronic design of NAO humanoid. In: IEEE international conference on robotics and automation, pp 769-774

60. Nalin M, Baroni I, Sanna A, Pozzi C (2012) Robotic companion for diabetic children: emotional and educational support to diabetic children, through an interactive robot. In: Proceedings of the 11th International Conference on Interaction Design and Children, pp 260-263

61. Shamsuddin S, Yussof H, Ismail L, Hanapiah FA, Mohamed S, Piah HA, Zahari NI (2012) Initial response of autistic children in humanrobot interaction therapy with humanoid robot NAO. In: IEEE 8th international colloquism on signal process and its Application, pp $188-193$

62. López Recio D, Márquez Segura E, Márquez Segura L, Waern A (2013) The NAO models for the elderly. In: Proceedings of the 8th $\mathrm{ACM} / \mathrm{IEEE}$ international conference on human-robot interaction, pp 187-188

63. Kim KJ, Park E, Shyam Sundar S (2013) Caregiving role in humanrobot interaction: a study of the mediating effects of perceived benefit and social presence. Comput Hum Behav 29:1799-1806

64. Shamsuddin S, Yussof H, Ismail LI, Mohamed S, Hanapiah FA, Zahari NI (2012) Initial response in HRI-a case study on evaluation of child with autism spectrum disorders interacting with a humanoid robot NAO. Procedia Eng 41:1448-1455

65. Kim A, Han J, Jung Y, Lee K (2013) The effects of familiarity and robot gesture on user acceptance of information. In: Human-robot interaction (HRI), 2013 8th ACM/IEEE international conference pp $159-160$

66. Pot E, Monceaux J, Gelin R, Maisonnier B (2009) Choregraphe: a graphical tool for humanoid robot programming. In: ProceedingsIEEE international workshop on robot and human interactive communication, pp 46-51

67. Kline P (2000) The handbook of psychological testing, 2nd edn. Routledge, New York

68. Heerink M (2011) Exploring the influence of age, gender, education and computer experience on robot acceptance by older adults. In: Human-robot interaction (HRI), 2011 6th ACM/IEEE international conference, pp 147-148

69. Mann HB, Whitney DR (1947) On a test of whether one of two random variables is stochastically larger than the other. Ann Math Stat 18(1):50-60
70. Hu PJ-H, Clark THK, Ma WW (2003) Examining technology acceptance by school teachers: a longitudinal study. Inf Manag 41(2):227-241

71. Bartneck C, Suzuki T, Kanda T, Nomura T (2007) The influence of people's culture and prior experiences with Aibo on their attitude towards robots. AI Soc 21:217-230

72. Baek Y, Jung J, Kim B (2008) What makes teachers use technology in the classroom? Exploring the factors affecting facilitation of technology with a Korean sample. Comput Educ 50(1):224-234

73. Shin D-H, Choo H (2011) Modeling the acceptance of socially interactive robotics: social presence in human-robot interaction. Interact Stud 12(3):430-460

74. Venkatesh V, Davis FD (2000) A theoretical extension of the technology acceptance model: four longitudinal field studies. Manage Sci 46:186-204

75. Rogers EM (2010) Diffusion of innovations. Simon and Schuster, New York

76. Mutlu B, Forlizzi J (2008) Robots in organizations? The role of workflow, social, and environmental factors in human-robot interaction. In: HRI ' 08 proceedings of the 3rd ACM/IEEE international conference on Human robot interaction, pp 287-294

77. Li D, Rau P, Li Y (2010) A cross-cultural study: effect of robot appearance and task. Int J Soc Robot 2:175-186

78. Dillon A (2002) Beyond usability: process, outcome and affect in human-computer interactions. Can J Inf Libr Sci 26:57

Daniela Conti graduated with BSc and MSc in Psychology and BSc in Psychiatric Rehabilitation and Social Education and received the Ph.D. in Neuroscience at University of Catania, Italy. In the academic years from 2013 to 2015 she was visiting at Plymouth University (UK), Centre for Robotics and Neural Systems. Currently she is Marie Curie Research Fellow at Sheffield Hallam University (UK). Her main research interests are focused on application of cognitive robotics to diagnosis of intellectual disability and psychiatric rehabilitation.

Santo Di Nuovo graduate in Philosophy and Psychology. Full Professor since 1990, now at University of Catania. Dean, Faculty of Education, now Department of Education, University of Catania, Italy. Vicepresident, National Conference of Academic Psychology. Main fields of research interest: Experimental Psychology-Artificial IntelligenceMethodology and assessment-Clinical-rehabilitative and Forensic Psychology. Author or co-author of about 380 books and papers.

Serafino Buono psychologist, Head of the Unit of Psychology at Oasi Institute for Research on Intellectual Disabilities and Brain aging, Troina (Italy). Author of a number of scientific papers in the field of Intellectual Disabilities, Learning Disabilities, Autism Spectrum Disorders, Neurodevelopmental Disorders. Co-editor of Life Span and Disability Journal.

Alessandro Di Nuovo received the MSc and Ph.D. degrees in computer engineering from the University of Catania, Italy, in 2005 and 2009, respectively. He is currently Senior Lecturer at Sheffield Hallam University (UK) and engaged in research activities in the University of Enna (Italy). His research specializes in computational intelligence and its application to cognitive modelling, human-robot interaction, computer-aided assessment of intellectual disabilities, and embedded computer systems. He is author of over 60 scientific articles and he was involved in many European research projects and networks. 